\title{
APROXIMACIÓN A LAS INVERSIONES EN INFRAESTRUCTURA DE LAS COMUNIDADES AUTÓNOMAS ESPAÑOLAS
}

Francisco Antonio García-Marquez

Universidad Nacional de Educación a Distancia

España 
Panorama Económico, Vol. 25 - No. 3 (Julio - Septiembre de 2017), pp. 311-332

Francisco Antonio García-Marquez

\title{
Aproximación a las inversiones en infraestructura de las Comunidades Autónomas Españolas
}

\begin{abstract}
Resumen
Las inversiones en infraestructura tienen un efecto muy notable en el desarrollo industrial, territorial y económico de regiones y naciones. Es por eso que en el presente trabajo mediante un análisis de conglomerados, se busca dar una aproximación a la evaluación de los efectos de esas inversiones en las economías de las Comunidades Autónomas españolas. Se consideran las variables de inversión en infraestructura por habitante, inversión en infraestructura por superficie, crecimiento del PIB per cápita, porcentaje medio de paro y número de empresas por cada 1000 habitantes de media, durante el periodo de crisis (2007-2013) y post-crisis (2014-2015), con el fin de identificar el papel fundamental que han tenido las inversiones en infraestructura en las economías regionales.

Palabras clave: inversiones en infraestructura, crisis económica, España, desarrollo territorial.

Clasificación JEL: O18, O11, E62, H54, R42
\end{abstract}

\section{An approach to infrastructure investments in the Spanish Autonomous Communities}

\begin{abstract}
Infrastructure investments have a significant effect on the industrial, territorial and economic development of regions and nations. Hence, in the present document, through cluster analysis an assessment of such effects on the Spanish Autonomous Communities is carried out. Variables such as infrastructure investmentper capita; infrastructure investment by areas; growth of GDP per capita, average unemployment rate, and number of enterprises per 1000 population on average during the crisis period (2007-2013) and the postcrisis period (2014-2015), to identify the fundamental role that infrastructure investments have played in the Spanish regional economies.

Keywords: Infrastructure investments, economic crisis, Spain, territorial development.
\end{abstract}

JEL Classification: O18, O11, E62, H54, R42

\section{Une approche aux investissements en infraestructure dans las communautés autonomes d'Espagne}

\footnotetext{
Résumé

Les investissements dans les infrastructures ont un impact très significatif sur le développement industriel, territorial et économique des régions et des nations. C'est pourquoi, dans le présent travail, nous allons effectuer une analyse par grappes. Cette analyse a pour but de donner une idée approximative de l'évaluation des effets de ces investissements sur les économies des communautés autonomes espagnoles. Tout cela à partir des variables: investissement dans les infrastructures par habitant, investissement dans les infrastructures par zone, croissance du PIB par habitant au cours de la période, pourcentage moyen de chômage sur la période et nombre d'entreprises pour 1000 habitants en moyenne. Nous travaillerons dans deux périodes différentes: 2007-2013 (période de crise) et 2014-2015 (période post-crise). L'objectif principal de ce travail est d'identifier le rôle fondamental que les investissements dans les infrastructures ont eu dans les économies régionales pendant ces années difficiles pour l'économie espagnole.

Mots-clés: Investissements dans les infrastructures, crise économique, Espagne, développement territorial. Nomenclature JEL: O18, O11, E62, H54, R42
} 


\section{Aproximación a las inversiones en infraestructura de las Comunidades Autónomas Españolas}

INFORMACIÓN DEL ARTÍCULO

Recepción de artículo: 08/04/2017

Concepto de evaluación: 25/05/2017

Aceptación de artículo: 15/06/2017
Francisco Antonio García-Marquez*

Universidad Nacional de Educación a Distancia

España

\section{INTRODUCCIÓN}

Sin duda, el desarrollo territorial está muy relacionado con las inversiones en infraestructura que realizan regiones y países. Esto es sobradamente conocido. El territorio, a lo largo del tiempo, debe evolucionar. Ya los primeros habitantes de nuestro mundo construían los primeros caminos abriéndose paso entre los matorrales de los bosques por los que debían circular. Más tarde, los romanos innovaron en infraestructura edificando maravillas como los acueductos, las calzadas, los anfiteatros o los teatros que, algunos de ellos, continúan parcialmente en pie en el momento presente. Los grandes castillos medievales, las embarcaciones que llevaron a los españoles al Nuevo Mundo, las líneas ferroviarias, los rascacielos o la misma llegada del hombre a la Luna son ejemplos que demuestran la importancia que tiene la inversión en infraestructura en el desarrollo del territorio. Si hace menos de 250 años, una persona tardaría unas 23 jornadas en trasladarse de Bilbao a Cádiz ${ }^{1}$, en el mundo actual, tecnológico y rápido, el mismo desplazamiento podría hacerse en apenas un par de horas. ¿Quién lo iba a decir?

El objetivo del presente artículo es realizar una aproximación a la evaluación de las inversiones en infraestructura llevadas a cabo por las Comunidades Autónomas (en adelante CCAA) de España. Se ha decidido hacer el estudio en ese territorio debido a la gran diversidad social, cultural, ideológica y económica de este país. España comprende el cuarto territorio más amplio de toda Europa con 505.957 kilómetros cuadrado, solo

\footnotetext{
* Autor para correspondencia 
superado por Francia, Ucrania y Rusia ${ }^{2}$; así mismo es la séptima nación europea en habitantes $(46.468 .102)^{3}$. Las lenguas habladas en España también hacen alusión a la diversidad territorial del país, teniendo un idioma oficial (el español) y cinco lenguas cooficiales (catalán, aranés, euskera, gallego y valenciano). Por último, se debe mencionar que la realidad económica de estas CCAA es ciertamente dispar, como se comprobará en los datos propuestos próximamente.

El periodo escogido para realizar el análisis es el 2007-2015, es decir, los años contemporáneos a la gran crisis económica y financiera que azotó a prácticamente todos los países del mundo. La Gran Recesión, que presentó sus primeros síntomas en agosto de 2007 y que, a duras penas, parece que se va dejando atrás, ha provocado grandes cambios en todos los indicadores económicos españoles. Desde el PIB per cápita, que se redujo de $24.300 €$ en 2008 a $22.518 €$ en $2013^{4}$, al porcentaje de desempleo, que no hizo más que aumentar entre los años 2007 y $2012^{5}$, los efectos de la misma han sido palpables en la realidad económico-social de España.

La naturaleza de las inversiones en infraestructura analizadas, en el presente artículo, es pública. De esta manera se pretende comprobar el esfuerzo inversor por parte de la Administración Pública de España y, su repercusión en las

2 Instituto Nacional de estadística (2017). [Acceso 2 de marzo de 2017]. http://www.ine.es/jaxi/Datos. htm?path=/t42/p01/10/\&file=110013b.px

3 EXPANSIÓN/datosmacro.com (junio de 2016). [Acceso el 2 de abril de 2017] Disponible en http:// www.datosmacro.com/demografia/poblacion/espana

4 EXPANSIÓN/datosmacro.com (2008-2013). [Acceso el 14 de marzo de 2017] Disponible en http://www. datosmacro.com/pib/espana

5 INE (2007-2015). [Acceso el 14 de marzo de 2017] Disponible en http://www.ine.es/jaxiT3/Datos. htm?t=4247 economías de las CCAA. Un esfuerzo, sin dudas, también de los sucesivos gobiernos estatales que han permitido conseguir hitos relevantes como disponer de la red viaria de alta capacidad más extensa de Europa, con más de 15.000 kilómetros o la segunda mayor red de alta velocidad ferroviaria del mundo con más de 2.500 kilómetros en servicio. ${ }^{6}$

\section{MARCO TEÓRICO.}

La relación entre inversiones en infraestructura y desarrollo territorial ha sido analizada por multitud de autores desde diversos puntos de vista. Aghón, Alburquerque y Cortés comentan en uno de sus escritos que la visión del desarrollo económico local se ha sumado a la reflexión sobre desarrollo económico en las últimas décadas. Se dan a conocer cuatro grandes ámbitos de análisis, entre los que se incluyen las infraestructuras facilitadoras del desarrollo, los servicios de apoyo a la producción y el fomento territorial de la innovación productiva y empresarial ${ }^{7}$. Por tanto, las inversiones en infraestructura marcan el devenir de las economías que las llevan a cabo, facilitando el desarrollo de los territorios nacionales, regionales, provinciales o rurales en las que se aplican.

El mismo Alburquerque, en otra de sus obras, toca un tema tan importante como actual: la evaluación del impacto ambiental. El autor comenta la importancia de las directivas comunitarias (97/11/CE) y las diferentes propuestas que se van publicando con el fin de conceder al tema de la contaminación y de la evaluación ambiental la importancia que deben tener.

6 CONVIVENCIA CÍVICA CATALANA (2016): Las inversiones en infraestructura en España. Análisis y distribución territorial. Estudio de la última década (2006-2015). Página 9.

7 AGHÓN, G.; ALBURQUERQUE F. Y CORTÉS P. (2001): Desarrollo económico local y descentralización en América Latina: un análisis comparativo. Página 12. 
Estas, en palabras del doctor, tienen por objeto garantizar la evaluación adecuada de las repercusiones sobre el medio ambiente de proyectos de infraestructuras ${ }^{8}$. El Libro Verde ${ }^{9}$ de la Unión Europea y el Libro $\mathrm{Azul}^{10}$ también plantean la importancia de combatir el cambio climático y desarrollar herramientas para concienciar a la población de la importancia de cuidar el entorno. Así pues, se comprueba que las inversiones en infraestructura tocan un tema transversal a muchos campos de estudio: el medioambiente. Hoy en día, la contaminación y el medioambiente son palabras que están en boca de muchos. Es sabido que se debe reciclar tanto como se pueda, conducir coches híbridos o eléctricos y utilizar energías renovables. Los consumidores, las empresas y los mismos gobiernos lo saben. A la hora de la planificación y la ejecución de las inversiones en infraestructura, provenga el dinero de la naturaleza de dónde provenga, se ha de realizar un estudio pormenorizado del impacto de esas inversiones en el medioambiente de los territorios.

Como se acaba de comentar, las inversiones en infraestructura, por lo general, suelen tener naturaleza pública, pero cada vez más, como escribe Sánchez Calderón, este dinero proviene de bolsillos privados. Añade el autor que desde mediados de los noventa, se ha venido estimulando el ingreso de la inversión privada en la construcción y gestión de la infraestructura, con lo cual se consolida la función de regulación del

8 ALBURQUERQUE, F. (2011): Planes de Desarrollo Sostenible en los Parques Naturales de Andalucía. Aproximación conceptual y metodológica. Página 25.

9 Libro verde de transporte y cambio climático (2010). Madrid: Colegio de Ingenieros de Caminos, Canales y Puertos.

10 FRITZ, J., \& HANUS, J. (2015). The European integrated maritime policy: The next five years.
Estado ${ }^{11}$. Lo público pasa a ocupar una posición de regulador más que de inversor. Eso es así, entre otras cosas, porque las inversiones en infraestructura vienen registrando niveles muy bajos tras la crisis del $2008^{12}$ y son las empresas privadas las que están llevando a cabo muchas de esas obras, de las que obtendrán posteriormente unos ingresos por el uso y disfrute de sus infraestructuras (carretas de peaje o edificios privados).

Pero, el Estado sigue gozando de un papel protagonista en lo que a inversiones en infraestructura se refiere. De hecho, Peña Sánchez, así lo recoge en una de sus obras cuando escribe que las inversiones que se están analizando en el presente artículo son uno de los instrumentos de política económica más directos de los que disponen los gobiernos modernos para influir tanto sobre el ritmo de crecimiento agregado como sobre la distribución geográfica de la actividad económica ${ }^{13}$. Por tanto, una buena inversión en infraestructura es un elemento fundamental para la creación de riqueza en cualquier territorio. Los gobiernos pueden influir, con su toma de decisiones, en el devenir de las regiones, en la distribución de la población o en los negocios de las empresas locales. Así mismo, las inversiones en infraestructura tienen efecto a priori y a posteriori en ese futuro de los territorios, puesto que tras

11 SÁNCHEZ CALDERÓN, F.V. (2008): Elementos para una geopolítica de los megaproyectos de infraestructura en América Latina y Colombia. Página 10.

12 GONZÁLEZ JULIÁN. La inversión en infraestructuras se convierte en enfermedad crónica. Capital Madrid (22 de Agosto de 2016). [Acceso el 1 de abril de 2017] Disponible en https://www. capitalmadrid.com/2016/8/22/43360/la-inversionen-infraestructuras-se-convierte-en-enfermedadcronica.html

13 PEÑA SÁNCHEZ, A. R. (2008): Las disparidades económicas regionales en España: Las infraestructuras como factor de convergencia en el periodo 19802000. Página 18. 
años de crecimiento económico se tiende a invertir más en infraestructura que en los años anteriores. Esto último provocó en España (tras el boom inmobiliario) grandes inversiones mal ejecutadas o planificadas que dieron lugar a aeropuertos desiertos, carreteras sin uso o edificios públicos que no desarrollan actividad alguna.

Sánchez Barquero es otro autor que ha escrito sobre esta relación entre inversiones en infraestructura y desarrollo territorial. En uno de sus trabajos demuestra que el aumento del gasto público en infraestructuras de transporte y comunicaciones, sea a través de las administraciones centrales o locales ${ }^{12}$ es una de las medidas que se adoptan para activar la economía real, estimulando la productividad y la competitividad. El profesor concluye que un aumento de recursos para mejorar las infraestructuras, con dinero público o privado, ayuda a la recuperación económica de regiones que arrastran problemas económicos o financieros. Por tanto, esta es una estrategia a seguir en tiempos de crisis. Además añade, en ese mismo escrito, que la mejora de las infraestructuras atrae actividades industriales y de servicios a las localidades y regiones rurales, lo que genera economías de diversidad y favorece el aumento de la productividad. Por tanto, para que exista generación o regeneración económica es indispensable una inversión adecuada en infraestructura y, esa inversión, repercutirá en la creación de nuevas industrias (y por tanto empleos) y en un aumento de la diversidad y la productividad dentro de los sectores empresariales territoriales.

Tras lo visto en los párrafos anteriores, se puede sacar como conclusión que una inversión adecuada en infraestructura ayuda a garantizar una mejora económica en el territorio en el que se lleve a cabo. Estas inversiones que, engloban a un conjunto enorme y dispar de elementos, ayudan, en gran medida, a que los ciudadanos de las regiones vivan mejor. $Y$ por mejor entendemos: se desplacen más rápido, tengan más posibilidades a la hora de escoger unos estudios, sea más fácil una pronta recuperación tras una enfermedad, que la luz eléctrica no falle o que el agua corriente sea de calidad.

\section{METODOLOGÍA.}

En el presente estudio se lleva a cabo el análisis de los comportamientos de las diferentes Comunidades Autónomas españolas en relación a las inversiones en infraestructura realizadas mediante la utilización de diferentes análisis de conglomerados en los periodos 2007-2013 y 2014-2015. La clasificación por conglomerados proporciona información del grado de similitud o discrepancia existente entre las variables estudiadas de cada CCAA.

Se realizarán cuatro análisis de conglomerados diferentes, dos en el periodo 2007-2013 y otros dos en el 2014-2015. Las variables utilizadas para realizar la parte metodológica en el análisis de conglomerados 1 son: inversión en infraestructura por habitante (€), crecimiento del PIB per cápita en el periodo, porcentaje medio de paro en el periodo ${ }^{14} \mathrm{y}$ número de empresas por cada 1000 habitantes de media en el periodo para el periodo 20072013. El análisis de conglomerados 3 estudia la relación entre las mismas variables que el análisis de conglomerados 1, pero en el periodo 2014-2015.

El análisis de conglomerados 2 se trabajará con las variables: inversión en infraestructura por superficie $(€)$, crecimiento del PIB per cápita en el periodo (\%), porcentaje me-

14 Datos obtenidos de Ministerio de Empleo y seguridad Social (MEYSS) (2017). [Acceso el 18 de febrero de 2017] Disponible en www.empleo.gob.es 
dio de paro en el periodo y número de empresas por cada 1000 habitantes de media en el periodo 2007-2013. El análisis de conglomerados 4 estudia la relación entre las mismas variables que el análisis de conglomerados 2, pero en el periodo 2014-2015. Como se puede observar, se sustituye, en estos dos últimos casos, la variable inversión en infraestructura por habitante por inversión en infraestructura por superficie. Se lleva a cabo esta distinción con la idea de analizar el impacto de la densidad de población de las CCAA en las inversiones realizadas en infraestructura, un apartado fundamental en el desarrollo de cualquier territorio.

El motivo por el que se escoge el periodo 2007-2013 como base de dos de los análisis de conglomerados realizados es el estudio del impacto de la crisis económica en las CCAA, más concretamente en el apartado de inversión en infraestructura. Se decide analizar también el periodo 2014-2015 con la intención de comprobar si la realidad de ligero crecimiento sufrido en ese tramo temporal, ha condicionado las inversiones llevadas a cabo en infraestructura.

La variable inversión en infraestructura por habitante resulta de la cantidad de inversión bruta en infraestructura ${ }^{15}$ de cada CCAA en los periodos de tiempo anteriormente señalados y los habitantes $^{16}$ que, por término medio, han estado empadronados en cada una de esas regiones en aquellos periodos. La inversión en infraestructura por superficie se calcula en función del territorio ${ }^{17}$,

15Datos obtenidos de la web del Ministerio de Fomento de España. Adjudicaciones de obra del Grupo Fomento (Años 2006 al 2015). [Acceso el 16 de febrero de 2017] Disponible en www.fomento.gob.es

16 Datos obtenidos de la web del Instituto Nacional de Estadística de España (2017). [Acceso el 16 de febrero de 2017] Disponible en www.ine.es

17 Datos obtenidos de la web del Instituto Nacional de Estadística de España (2017). [Acceso el 18 de febrero de 2017]. www.ine.es en kilómetros cuadrados, de las CCAA españolas.

La variable crecimiento del PIB per cápita ha sido calculada utilizando los datos de PIB $^{18}$ de los años 2007 y 2013 y de 2014 y 2015, respectivamente. Debido a la crisis económica y financiera que dio comienzo en los años 2007/2008, los resultados del PIB en el primer periodo son negativos en todas las CCAA. Sin embargo el crecimiento del PIB tiene valores positivos en cada CCAA en el segundo periodo analizado. Esto último es debido, sin duda, a la pequeña aceleración económica sufrida tras el periodo de crisis.

La variable número de empresas por cada 1000 habitantes ${ }^{19}$ se ha obtenido realizando la media de empresas existentes para el periodo que se estudian. Así mismo, este artículo se ha realizado analizando las variables de todas las CCAA a excepción de las Ciudades Autónomas de Ceuta y Melilla por las particularidades que presentan ambas ciudades. Además, para llevar a cabo el análisis de conglomerados se ha empleado el método del vecino más cercano.

En futuros trabajos podría plantearse la inclusión de otros indicadores económicos o socio-demográficos que muestren, desde otro enfoque, la realidad de las CCAA españolas. Algunos ejemplos pueden ser el gasto público, el porcentaje de inmigrantes en un territorio, el déficit público o el gasto de las economías de esos territorios por sectores productivos.

18 Datos obtenidos de la web del Instituto Nacional de Estadística de España (2017). [Acceso el 18 de febrero de 2017]. www.ine.es

19 Datos obtenidos del Directorio Central de Empresas (DIRCE) disponible en el INE. [Acceso el 14 de febrero de 2017] http://www.ine.es/jaxi/menu. do?type=pcaxis $\&$ path $=/$ t37/p201/\&file=inebase 
Tabla 1 Datos CCAA 2007-2013.

\begin{tabular}{|c|c|c|c|c|c|c|}
\hline COMONIDADES AUTÓNOMAS & $\begin{array}{l}\text { Inversión } \\
\text { Inf raest. } \\
2007 / 2013\end{array}$ & $\begin{array}{c}\text { Crecimiento PIB } \\
2007 / 2013\end{array}$ & $\begin{array}{c}\text { Porcentaje paro } \\
2007 / 2013\end{array}$ & $\begin{array}{l}\text { Inv. Inf raest. } \\
\text { pc } 2007 / 2013\end{array}$ & $\begin{array}{l}\text { Inv. Inf raest. } \\
\text { km2 2007/2013 }\end{array}$ & $\begin{array}{c}\mathrm{H}^{\circ} \\
\text { empresas } / 1000 \mathrm{hb} \\
2007 / 2013\end{array}$ \\
\hline Andalucía & $6,46442 \mathrm{E} 9$ & $-10,49$ & 26,31 & 776,84 & 73829 & 59,9 \\
\hline Aragón & 1226730000 & $-6,38$ & 13,96 & 917,52 & 25707 & 68,45 \\
\hline Asturias & 791561000 & $-10,42$ & 15,72 & 733,74 & 74647 & 65,33 \\
\hline Baleares & 535810000 & $-11,19$ & 17,59 & 490,34 & 107334 & 81,56 \\
\hline Canarias & 847365000 & $-10,4$ & 25,41 & 403,83 & 113786 & 64,85 \\
\hline Cantabria & 596103000 & $-10,96$ & 13,19 & 1013,95 & 112028 & 66,28 \\
\hline Castilla La Mancha & 1395883000 & $-8,5$ & 20,15 & 672,1 & 17567 & 63,22 \\
\hline Castilla León & $4,56488 \mathrm{~B} 9$ & $-5,85$ & 14,99 & 1791,84 & 48447 & 65,99 \\
\hline Cataluña & $6,76938 \mathrm{E} 9$ & $-7,2$ & 16,29 & 907,31 & 210799 & 81,23 \\
\hline Comunidad Valenciana & $3,06654 \mathrm{E} 9$ & $-10,67$ & 20,51 & 604,99 & 131866 & 70,14 \\
\hline Extremadura & 982299000 & $-4,77$ & 23,42 & 890,82 & 23594 & 59,59 \\
\hline Galicia & 4,67241E9 & $-4,36$ & 14,83 & 1677,85 & 157985 & 71,18 \\
\hline La Rioja & 307445000 & $-6,19$ & 14,04 & 961,12 & 60941 & 72,15 \\
\hline Madrid & 1945133000 & $-4,1$ & 14,17 & 304,73 & 242294 & 79,1 \\
\hline Murcia & 622263000 & $-8,44$ & 20,68 & 429,43 & 54999 & 64,27 \\
\hline Navarra & 53826000 & $-6,97$ & 11,62 & 85,15 & 5180 & 66,97 \\
\hline País Vasco & 1208719000 & $-4,83$ & 11,34 & 555,96 & 167089 & 76,21 \\
\hline
\end{tabular}

Fuente: Elaboración propia con datos recogidos de los organismos antes citados.

Tabla 2 Datos CCAA 2014-2015.

\begin{tabular}{|c|c|c|c|c|c|c|}
\hline COUUNIDADES AUTÚNOUAS & $\begin{array}{l}\text { Inversión } \\
\text { Infraest. } \\
2014 / 2015\end{array}$ & $\begin{array}{c}\text { Crecimiento PIB } \\
2014 / 2015\end{array}$ & $\begin{array}{c}\text { Porcentaje paro } \\
2014 / 2015\end{array}$ & $\begin{array}{l}\text { Inv. Infraest. } \\
\text { pc } 2014 / 2015\end{array}$ & $\begin{array}{l}\text { Inv. Inf raest. } \\
\text { km2 2014/2015 }\end{array}$ & $\begin{array}{c}\mathrm{H}^{\circ} \\
\text { empresas } / 1000 \mathrm{hb} \\
2014 / 2015\end{array}$ \\
\hline Andalucía & 584450000 & 3,2 & 33,16 & 69,57 & 6674,93 & 56,48 \\
\hline Aragón & 9605000 & 2,7 & 18,23 & 7,27 & 201,28 & 67,34 \\
\hline Asturias & 81982000 & 3,1 & 20,12 & 77,6 & 7731,23 & 63,32 \\
\hline Baleares & 85306000 & 3,2 & 18,78 & 77,27 & 17088,5 & 77,62 \\
\hline Canarias & 121530000 & 2,8 & 30,76 & 57,8 & 16319,3 & 62,16 \\
\hline Cantabria & 40929000 & 2,6 & 18,53 & 69,74 & 7691,98 & 63,07 \\
\hline Castilla La Mancha & 9088000 & 3,2 & 27,67 & 4,39 & 114,37 & 60,15 \\
\hline Castilla León & 256984000 & 2,9 & 19,51 & 103,48 & 2727,37 & 64,55 \\
\hline Cataluña & 427639000 & 3,3 & 19,46 & 56,92 & 13316,7 & 77,26 \\
\hline Comunidad Valenciana & 159486000 & 3,6 & 24,3 & 31,94 & 6858,14 & 67,14 \\
\hline Extremadura & 122780000 & 3 & 29,44 & 111,99 & 2949,03 & 58,11 \\
\hline Galicia & 299666000 & 3,2 & 20,48 & 109,35 & 10132,4 & 70,67 \\
\hline La Rioja & 19493000 & 2,8 & 16,77 & 61,29 & 3863,83 & 71,37 \\
\hline Madrid & 222261000 & 3,4 & 17,9 & 34,48 & 27685,7 & 77,81 \\
\hline Murcia & 148141000 & 3,1 & 25,6 & 100,98 & 13093,6 & 60,26 \\
\hline Navarra & 2633000 & 2,9 & 14,77 & 4,11 & 253,39 & 66,12 \\
\hline País Vasco & 399878000 & 3,1 & 15,55 & 182,67 & 55277,6 & 68,02 \\
\hline
\end{tabular}

Fuente: Elaboración propia con datos recogidos de los organismos antes citados.

\section{RESULTADOS.}

Con la ayuda de los resultados alcanzados, en cada uno de los cuatro análisis de conglomerados elaborados, y del estudio de la realidad económica de cada CCAA, a partir del análisis de los datos de las variables utilizadas para la elaboración de los conglomerados, se dividirán a las CCAA en varios grupos.

A continuación se muestra cada uno de los resultados obtenidos tras la realización de los análisis de conglomerados efectuados y las clasificaciones de las CCAA llevadas a cabo en cada caso. 
Análisis de conglomerados 1. Clúster que combina las variables: inversión en infraestructura per cápita, crecimiento del PIB, número medio de empresas por cada 1000 habitantes y porcentaje de paro medio en el periodo 2007-2013 en las CCAA de España.

Tabla 3 Resumen de Conglomeración y centroides del análisis de conglomerados 1.

\begin{tabular}{|c|c|c|c|c|}
\hline \multicolumn{3}{|c|}{ Resumen de Conglomeración } & & \\
\hline Conglomerado & Miembras & Porcentaje & & \\
\hline 1 & 12 & 70,59 & & \\
\hline 2 & 1 & 5,88 & & \\
\hline 3 & 2 & 11,76 & & \\
\hline 4 & 1 & 5,88 & & \\
\hline 5 & 1 & 5,88 & & \\
\hline \multicolumn{5}{|l|}{ Centroides } \\
\hline Conglomerado & \multicolumn{2}{|c|}{ Crecimienio PIB 2007/2013 } & Inv. Infraest.pe 2007/2013 & $N^{0}$ empresas $/ 1000 \mathrm{hb} 2007 / 2013$ \\
\hline 1 & \multicolumn{2}{|c|}{$-8,215$} & 690,127 & 69,2608 \\
\hline 2 & \multicolumn{2}{|l|}{$-11,19$} & 490,34 & 81,56 \\
\hline 3 & \multicolumn{2}{|l|}{$-5,105$} & 1734,84 & 68,585 \\
\hline 4 & \multicolumn{2}{|l|}{$-4,77$} & 890,82 & 59,59 \\
\hline 5 & \multicolumn{2}{|l|}{$-6,97$} & 85,15 & 66,97 \\
\hline Conglomerado & \multicolumn{2}{|c|}{ Porcentaje paro 2007/2013 } & & \\
\hline 1 & \multicolumn{2}{|l|}{17,6475} & & \\
\hline 2 & \multicolumn{2}{|l|}{17,59} & & \\
\hline 3 & \multicolumn{2}{|l|}{14,91} & & \\
\hline 4 & \multicolumn{2}{|l|}{23,42} & & \\
\hline 5 & \multicolumn{2}{|l|}{11,62} & & \\
\hline
\end{tabular}

Fuente: Elaboración propia

Gráfica 1 Dendograma 1 resultante del análisis de conglomerados 1.

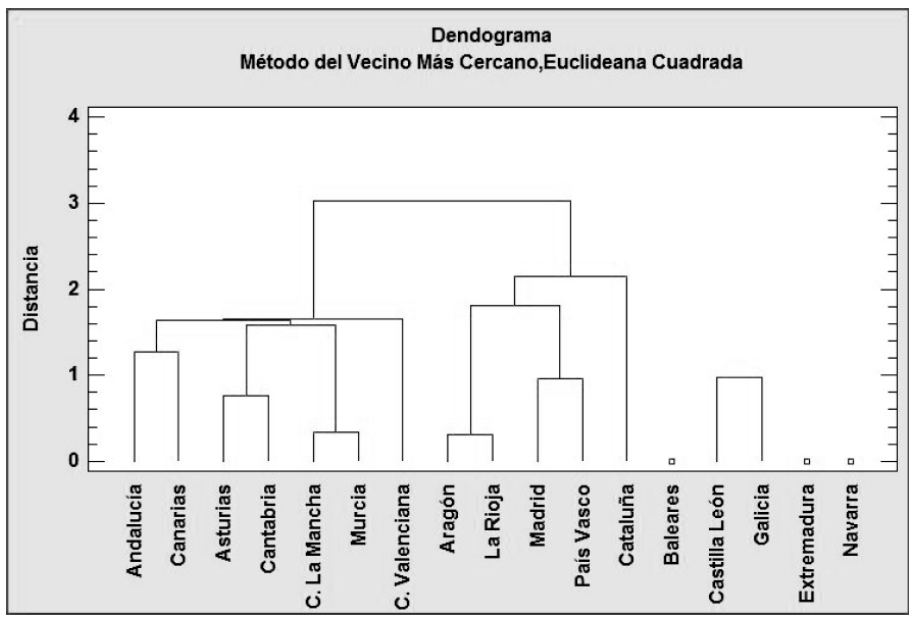

Fuente: Elaboración propia. 
Se han dividido, en este primer caso, a las CCAA en siete grupos. Para que sean más claras y visibles las explicaciones que se darán a continuación, es necesario repasar cuáles fueron los niveles medios de las variables analizadas en el periodo concreto. La inversión en infraestructura media por habitante en las CCAA españolas fue de $777,5 €$. El crecimiento del PIB medio por Comunidad, evidentemente negativo por motivo de la crisis, tuvo un promedio de $-7,75 \%$. La media de empresas por cada 1000 habitantes en las regiones fue del 69,2 y la tasa de paro media por Comunidad se mantuvo en el 17,31\%:

1. Territorios con situación económica favorable y niveles de inversión en infraestructura por habitante medio-altos: este primer grupo de Comunidades está formado por Aragón, La Rioja, Madrid, País Vasco y Cataluña. Estas regiones, que han tenido un crecimiento del PIB más alto que la media del resto de Comunidades, muestran niveles de inversión en infraestructura por habitantes, tasa de desempleo y número de empresas por cada 1000 habitantes similares. Entre los datos numéricos que demuestran que son economías fuertes destacan, por ejemplo, los de desempleo, puesto que la media de las Comunidades españolas en esta variable sobrepasa el $17 \%$ y, en este grupo, solo Cataluña supera el 15\% (16,29\%). Así mismo su crecimiento del PIB, aunque decreciente, oscila entre el $-4,1$ de Madrid y el -7,2 de Cataluña. Históricamente estas regiones son zonas con una industria fuerte y un sector servicios punteros en el país. A excepción de Madrid, que se queda muy alejado de la media en inversión en infraestructura $(472,77 €$ para ser exactos), el resto de estas CCAA invierten valores cercanos a la media.

2. Territorios con situación económica favorable y niveles de inversión en infraestructura por habitante bajos: en este grupo únicamente se incluye a la Comunidad Foral de Navarra. Los datos que se manejan la unen estrechamente al primer grupo analizado, pero su inversión en infraestructura por habitante en francamente escasa, superando apenas los $85 €$. Es decir, destina a la inversión en infraestructura un 11\% de la media de las CCAA españolas.

3. Territorios con nivel económico medio y niveles muy elevados en inversión en infraestructura por habitante: formado por Castilla León y Galicia. Los datos de las variables tomadas identifican a estas regiones con la media, aunque, bien es cierto que, los niveles de crecimiento del PIB y tasa de paro quedan por debajo de esta. Son particularmente interesantes los datos tan elevados de inversión en infraestructura que presentan estas regiones (un 223\% más que la media de las Comunidades de España). En el caso de Castilla León, sin duda, se debe a la muy baja densidad de población existente en la región (junto con Extremadura y Castilla-La Mancha presentan las tasas más bajas de España con 26 habitantes por kilómetro cuadrado al final del periodo estudiado) y a su superficie, puesto que es la CCAA con mayor territorio. En el caso de Galicia, esto es debido a los múltiples proyectos llevados en el área de infraestructura, entre los que destacan la inversión en red eléctrica y ferrocarril entre otros. 
4. Territorios con nivel económico medio-bajo, baja tasa de inversión en infraestructura por habitante y grandes niveles de emprendimiento: los datos recogidos acercan a Baleares, única componente del conglomerado 3 , al grupo anteriormente mencionado. Sin embargo cuenta con una diferencia muy importante: el número de empresas por cada 1000 habitantes. Este es de $81 ` 56$, siendo el dato más alto de toda España, en buena parte debido al grandísimo negocio turístico establecido en la zona.

5. Territorios con situación económica baja y niveles de inversión en infraestructura por habitante bajos: este grupo está compuesto por cinco miembros: Asturias, Cantabria, Castilla-La Mancha, Murcia y la Comunidad Valenciana. A pesar de encontrarse en un mismo conglomerado, sus economías cuentan con realidades dispares puesto que, aunque tengan niveles muy parejos en crecimiento del PIB y número de empresas por número de habitantes, la tasa de desempleo de Asturias y Cantabria es mucho más baja que la de las otras CCAA (entre 5 y 7 puntos menos). Sus inversiones en infraestructura no sobrepasan en ningún caso la media de las CCAA de la nación.

6. Territorios con situación económica muy baja, nivel de inversión en infraestructura altos y alto crecimiento económico: formado exclusivamente por Extremadura. Esta CCAA cuenta con los mejores datos de crecimiento del PIB, pues solo han decrecido un $4,77 \%$. Su gasto en infraestructura y su tasa de paro es bastante mayor a la media. Así mismo, el ratio de empresas por cada 1000 habitantes es bastante menor que el de la media de la CCAA españolas. A priori, se podría definir como una economía estable, con negocios que no han notado tanto la crisis como en otros lugares de la península, y que ha decidido invertir más en infraestructura para intentar paliar las altas tasas de desempleo en la zona.

7. Territorios con situación económica muy baja y tasas medio-bajas de inversión en infraestructura por habitante: grupo formado por Andalucía y Canarias. Es cierto que estas CCAA se encuentran dentro del clúster anterior, pero los datos que se manejan arrojan a la luz que su situación económica y financiera es más delicada que la de las CCAA vecinas. Crecimiento del PIB muy bajo, altísimas tasa de paro (las mayores de España), bajo número de empresas por 1000 habitantes e inversión en infraestructura per cápita baja; esta es la realidad de estas dos regiones.

Se comprueba, por tanto, las grandes diferencias existentes entre las variables analizadas en las CCAA españolas. Los resultados obtenidos son negativos en todas las áreas de estudio y esto es provocado, sin duda, por la gran crisis del 2007-2008.

Análisis de conglomerados 2. Clúster que combina las variables: inversión en infraestructura por kilómetro cuadrado, crecimiento del PIB, número medio de empresas por cada 1000 habitantes y porcentaje de paro medio en el periodo 2007-2013 en las CCAA de España. 
Tabla 4 Resumen de Conglomeración y centroides del análisis de conglomerados 2 .

Resumen de Conglomeración
\begin{tabular}{||l|l|l|}
\hline Conglomerado & Miembros & Porcentaje \\
\hline 1 & 7 & 41,18 \\
\hline 2 & 4 & 23,53 \\
\hline 3 & 1 & 5,88 \\
\hline 4 & 4 & 23,53 \\
\hline 5 & 1 & 5,88 \\
\hline
\end{tabular}

\section{Centroides}

\begin{tabular}{||l|l|l|}
\hline Conglomerado & Crecimiento PIB 2007/2013 & Inv. Infraest. $\mathrm{km} 2$ 2007/2013 \\
\hline 1 & $-9,98286$ & 82674,6 \\
\hline 2 & $-6,3475$ & 35068,8 \\
\hline 3 & $-11,19$ & 107334 \\
\hline 4 & $-5,1225$ & 194542, \\
\hline 5 & $-4,77$ & 23594,0 \\
\hline
\end{tabular}

\begin{tabular}{||l|l|l|}
\hline Conglomerado & $N^{*}$ empresas/1000hb 2007/2013 & Porcentaje paro 2007/2013 \\
\hline 1 & 64,8557 & 20,2814 \\
\hline 2 & 68,39 & 13,6525 \\
\hline 3 & 81,56 & 17,59 \\
\hline 4 & 76,93 & 14,1575 \\
\hline 5 & 59,59 & 23,42 \\
\hline
\end{tabular}

Fuente: Elaboración propia.

Gráfica 2 Dendograma resultante del análisis de conglomerados 2 .

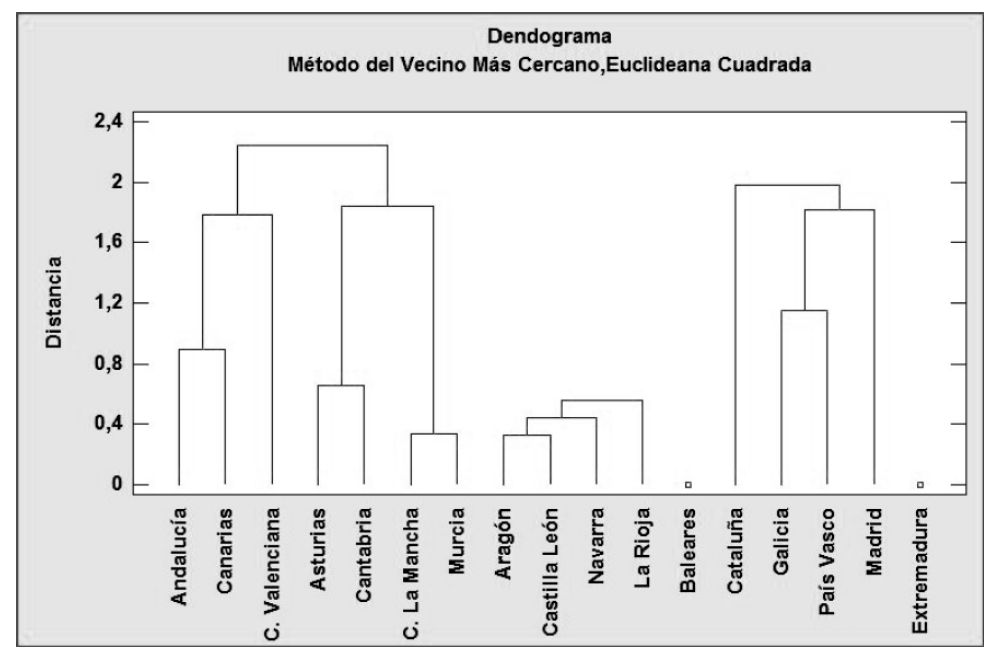

Fuente: Elaboración propia. 
Se divide en seis grupos a las CCAA españolas en este caso. Se debe conocer para entender las clasificaciones realizadas que la inversión media en infraestructura en las CCAA de España es de 95.770,12 $€$ por kilómetro cuadrado.

6. Territorios con situación económica muy baja y niveles de inversión en infraestructura por superficie medioaltos: compuesto por Andalucía y Canarias. Tienen tasas de empleo muy superiores a la media de las CCAA españolas. Su crecimiento del PIB es mucho más bajo que la media española (casi 3 puntos) y su nivel de empresas por 1000 habitantes es, también, inferior a la media. Sin embargo el nivel de inversión en infraestructura por extensión es ligeramente mayor a la media.
Tras el análisis de los conglomerados llevado a cabo, se comprueba que la densidad de población tiene un papel fundamental a la hora de clasificar a las CCAA en función de sus niveles de inversión en infraestructura. Las regiones con situaciones económicas parejas, a nivel nacional, tienden a invertir niveles similares en infraestructura en función de su superficie y no en función de sus habitantes. Por tanto, el análisis clúster 2 proporciona información más exacta de la relación económica entre las CCAA españolas y esas inversiones.

Análisis de conglomerados 3. Clúster que combina las variables: inversión en infraestructura per cápita, crecimiento del PIB, número medio de empresas por cada 1000 habitantes y porcentaje de paro medio en el periodo 2014-2015 en las CCAA de España.

Tabla 5 Resumen de Conglomeración y centroides del análisis de conglomerados 3.

\begin{tabular}{|c|c|c|c|c|}
\hline \multicolumn{3}{|c|}{ Resumen de Conglomeración } & & \\
\hline Conglamerado & Miembras & Porcentaje & & \\
\hline 1 & 13 & 76,47 & & \\
\hline 2 & 1 & 5,88 & & \\
\hline 3 & 1 & 5,88 & & \\
\hline 4 & 1 & 5,88 & & \\
\hline 5 & 1 & 5,88 & & \\
\hline \multicolumn{5}{|l|}{ Centroides } \\
\hline Conglomerado & \multicolumn{2}{|c|}{ Crecimiento PIB 2014/2015 } & In. Infrast. pe $2014 / 2015$ & $N^{*}$ empresas/1000ho 2014/2015 \\
\hline 1 & \multicolumn{2}{|c|}{3,03077} & 68,0038 & 67,2292 \\
\hline 2 & \multicolumn{2}{|l|}{2,8} & 57,8 & 62,16 \\
\hline 3 & \multicolumn{2}{|l|}{3,2} & 4,39 & 60,15 \\
\hline 4 & \multicolumn{2}{|l|}{3,6} & 31,94 & 67,14 \\
\hline 5 & \multicolumn{2}{|l|}{3,1} & 182,67 & 68,02 \\
\hline Conglomerado & \multicolumn{2}{|c|}{ Porcentage paro 2014/2015 } & & \\
\hline 1 & \multicolumn{2}{|l|}{20,9808} & & \\
\hline 2 & \multicolumn{2}{|l|}{30,76} & & \\
\hline 3 & \multicolumn{2}{|l|}{27,67} & & \\
\hline 4 & \multicolumn{2}{|l|}{$24, \hat{3}$} & & \\
\hline 5 & \multicolumn{2}{|l|}{15,55} & & \\
\hline
\end{tabular}

Fuente: Elaboración propia. 


\section{Gráfica 3 Dendograma resultante del análisis de conglomerados 3}

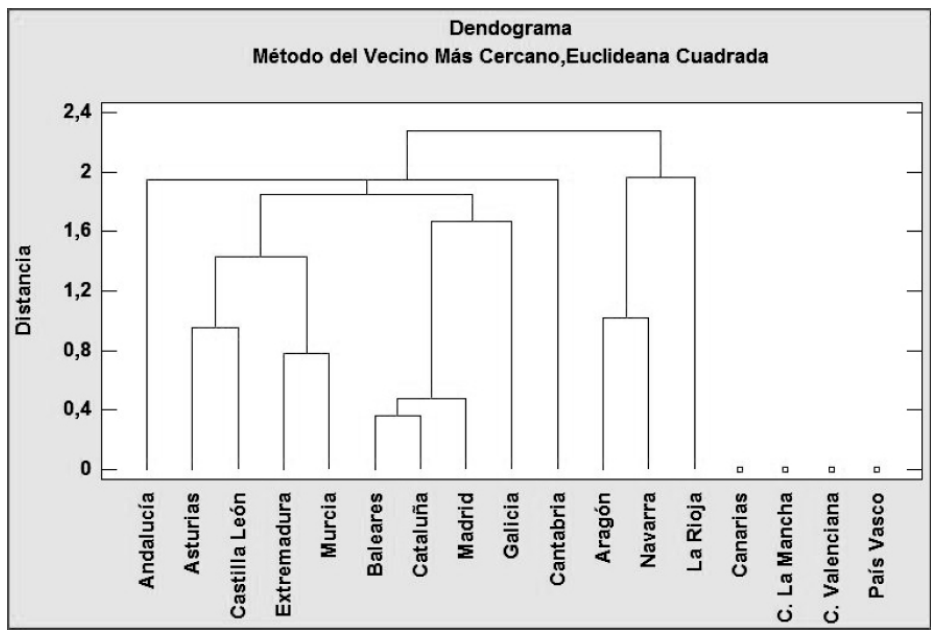

Fuente: Elaboración propia.

El tercer análisis clúster que se presenta en el artículo, se lleva a cabo en un periodo post crisis (2014-2015). Naturalmente los datos de las variables utilizadas están claramente marcados por esa realidad que, poco a poco, se va dejando atrás: el paro medio nacional en el periodo es muy alto $(21,83)$, el crecimiento del PIB es, aunque creciente, aún bajo $(3,06 \%)$ y el nivel de empresas por 1000 habitantes es algo más bajo que en el periodo anterior. Los niveles de inversión media en infraestructura por habitante son francamente bajos en comparación con el anterior periodo $(68,28 €)$. Siete son las clasificaciones en las que se dividen las situaciones vividas en aquella etapa en las CCAA españolas:

1. Territorios con situación económica favorable y con niveles de inversión en infraestructura muy altos: está compuesto únicamente por el País Vasco. Con un número de empresas por 1000 habitantes y un crecimiento del PIB en torno a la media, su tasa de desempleo es muy inferior a cualquier otra (6 puntos menos) y la inversión en infraestructura media de sus habitantes se eleva a los 182'67€, es decir, casi triplica la media.

2. Territorios con situación económica favorable y con niveles de inversión en infraestructura medios: formado por Baleares, Cataluña, Madrid y Galicia. Los datos que se manejan de estas CCAA son similares en crecimiento del PIB y número de empresas por cada 1000 habitantes (mayores a la media de las CCAA), tasa de paro inferior a la de la media $\mathrm{y}$ datos de inversión en infraestructura que rondan la media de las regiones españolas.

3. Territorios con nivel económico medio y baja tasa de inversión en infraestructura: compuestos por Aragón, Navarra y La Rioja. Teniendo un crecimiento del PIB en el periodo algo más bajo que la media, un ratio de 
empresas por 1000 habitantes similar a la media y, eso sí, niveles de tasa de desempleo ciertamente inferiores a la media (en torno a 5,3 puntos menos), estas regiones apenas invierten en infraestructura por habitante (solo Aragón supera el $10 \%$ de la media de las CCAA).

4. Territorios con situación económica media y niveles de inversión en infraestructura por habitante medio-altos: este nuevo grupo está formado por las CCAA de Cantabria, Asturias y Castilla León. Cuentan con porcentajes similares a la media española en crecimiento del PIB y en el número de empresas por cada 1000 habitantes. Así mismo su inversión en infraestructura por habitante es mayor que la media. Los niveles de desempleo son más bajos que la media de las CCAA.

5. Territorios con situación económica baja y niveles de inversión en infraestructura por habitante altos: este grupo está formado por Extremadura y Murcia. Cuentan con niveles de desempleo altos, baja tasa de empresas por 1000 habitantes, crecimiento del PIB medio y, eso sí, un nivel de inversión en infraestructura que sobrepasa la media de las CCAA del Estado Español.

6. Territorios con situación económica baja y niveles de inversión en infraestructura por habitante bajos: en él quedan incluidas las CCAA de Castilla-La Mancha y Valencia. Aunque el nivel de empresas por cada 1000 habitantes en el caso de la Comunidad Valenciana supera la media, el resto de datos de las variables estudiadas son muy parecidos al del grupo antes citado. La gran salvedad es que el gasto que hacen en inversión en infraestructura es francamente bajo, resaltando el caso de Castilla-La Mancha que es apenas algo mayor del $6 \%$ de la media de las Comunidades españolas.

7. Territorios con situación económica muy baja y niveles de inversión en infraestructura por habitante medios: Andalucía y Canarias. Tienen niveles de desempleo muy altos ( 9 puntos más que la media), crecimiento del PIB y número de empresas por debajo de la media y niveles de inversión algo más bajos, también.

Tras el análisis clúster de estas variables se comprueba que CCAA con una situación económica tan dispar como Cataluña o Andalucía, se encuentran dentro del mismo conglomerado. Estas claves ayudan a seguir dando pasos hacia la conclusión de que las CCAA con situación económica parecida, invierten también, niveles homogéneos en infraestructura en función del territorio que ocupan y no de la población que ostentan. Así mismo, los resultados reflejan que aquellas CCAA que han sentido menos la última crisis (País Vasco o Madrid), invierten más en infraestructura que en aquellas otras donde la crisis ha provocado efectos más negativos (Andalucía o Canarias).

Análisis de conglomerados 4. Clúster que combina las variables: inversión en infraestructura por kilómetro cuadrado, crecimiento del PIB, número medio de empresas por cada 1000 habitantes y porcentaje de paro medio en el periodo 20014-2015 en las CCAA de España. 
Tabla 6 Resumen de Conglomeración y centroides del análisis de conglomerados 4.

\begin{tabular}{|l|l|l|}
\hline \multicolumn{4}{|l|}{ Resumen de Conglomeración } \\
\hline Conglomerado & Miembros & Porcentaje \\
\hline 1 & 10 & 58,82 \\
\hline 2 & 4 & 23,53 \\
\hline 3 & 1 & 5,88 \\
\hline 4 & 1 & 5,88 \\
\hline 5 & 1 & 5,88 \\
\hline
\end{tabular}

\section{Centroides}

\begin{tabular}{||l|l|l|}
\hline Conglomerado & Crecimiento PIB 2014/2015 & Inv. Infraest. $\mathrm{km} 2$ 2014/2015 \\
\hline 1 & 2,95 & 4530,1 \\
\hline 2 & 3,275 & 17055,8 \\
\hline 3 & 2,8 & 16319,3 \\
\hline 4 & 3,6 & 6858,14 \\
\hline 5 & 3,1 & 55277,6 \\
\hline
\end{tabular}

\begin{tabular}{|l|l|l|}
\hline Conglomerado & $N^{0}$ empresas/1000hb 2014/2015 & Porcentaje paro 2014/2015 \\
\hline 1 & 63,077 & 22,38 \\
\hline 2 & 75,84 & 19,155 \\
\hline 3 & 62,16 & 30,76 \\
\hline 4 & 67,14 & 24,3 \\
\hline 5 & 68,02 & 15,55 \\
\hline
\end{tabular}

Fuente: Elaboración propia.

\section{Gráfica 4 Dendograma resultante del análisis} de conglomerados 4 .

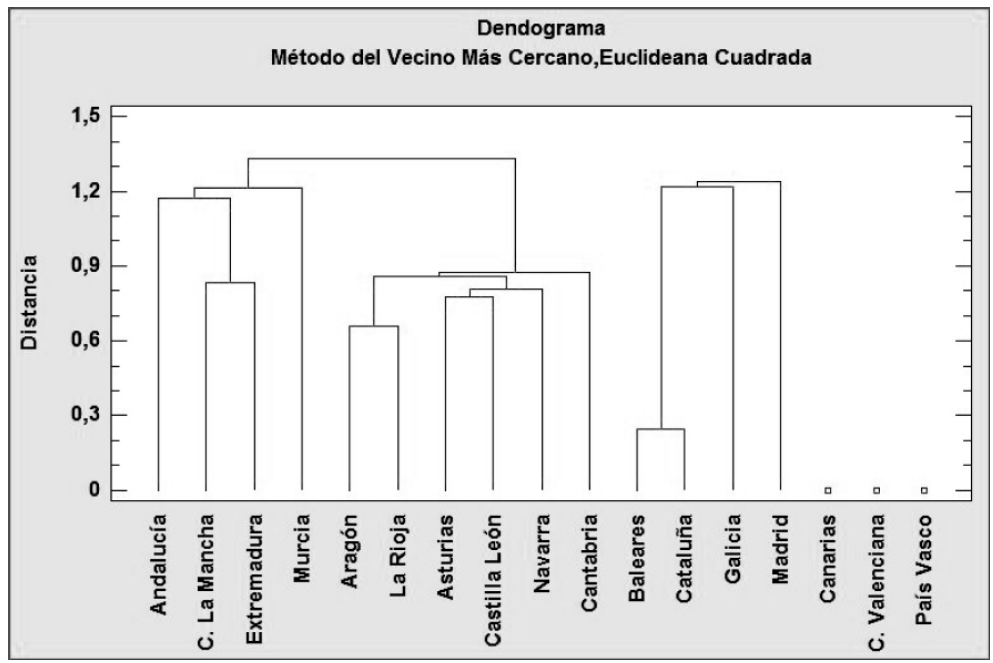

Fuente: Elaboración propia. 
Para analizar los resultados que aporta el clúster es necesario conocer que la media de inversión de las CCAA españolas por superficie es de 11.292,90€. La clasificación realizada divide, en este caso, a las regiones en siete grupos:

1. Territorios con situación económica favorable y con niveles de inversión en infraestructura muy altos: al igual que ocurriese en el anterior análisis de conglomerados 3, el País Vasco se cuela como único integrante de este grupo. Casi llega a quintuplicar la cantidad invertida en infraestructura por superficie de la media de las CCAA. Además, queda definida como una economía fuerte por su bajo nivel de desempleo, su crecimiento medio del PIB y su nivel de empresas por 1000 habitantes superior a la media.

2. Territorios con situación económica favorable y con niveles de inversión en infraestructura por superficie medioaltos: formado por Baleares, Cataluña, Galicia y Madrid. Cuentan con un crecimiento más alto que la media y su número de empresas por 1000 habitantes es muy superior a la media española. Sin embargo las tasas de desempleo son claramente inferiores. Sus niveles de inversión por kilómetros cuadrado son superiores a la de la media. De momento, los resultados obtenidos con estas nuevas variables son muy parecidos al anterior análisis de conglomerados.

3. Territorios con situación económica media y niveles de inversión en infraestructura por superficie bajos: Aragón, La Rioja, Asturias, Castilla León, Navarra y Cantabria componen el segundo conglomerado. Los datos muestran en estas CCAA, niveles de crecimiento medios, número bajo de empresas y porcentajes de desempleo muy elevados. Así mismo, los niveles de inversión en infraestructura por metro cuadrado son prácticamente la tercera parte de la media española.

4. Territorios con situación económica baja y niveles de inversión en infraestructura por superficie bajos: compuesto por Castilla-La Mancha y Extremadura. Su situación económica es delicada, pues sus niveles de desempleo son altos y su crecimiento del PIB en el periodo es medio. Así mismo el ratio empresas por 1000 habitantes es más bajo que la media de las CCAA. Además estas dos regiones presentan unos niveles de inversión por superficie francamente bajos. Curiosamente estas dos CCAA, como se ha visto anteriormente, cuentan con los niveles de densidad de población más bajos de España.

5. Territorios con situación económica baja y niveles de inversión en infraestructura por superficie medio: Comunidad Valenciana y la Región de Murcia quedan inmersas dentro de este grupo que presenta datos muy parecidos al anterior conglomerado, pero cuenta con unos niveles de inversión por superficie más cercanos a la media. La gran diferencia entre esta dos CCAA es su número de empresas por 1000 habitantes, que es superior a la media en el caso de la Comunidad Valenciana y más baja en el caso de Murcia.

6. Territorios con situación económica muy baja y niveles de inversión en infraestructura por superficie altos: Canarias, al igual que en el caso anterior, forma un único conglomerado. Al igual 
que se comentó en el análisis clúster 3, esta región presenta una situación económica baja e invierte una buena parte de su riqueza en la infraestructura.

7. Territorios con situación económica muy baja y niveles de inversión en infraestructura por superficie bajos: compuesto únicamente por Andalucía. A priori, esta economía tiene una situación económica muy difícil. Esto es provocado por su alta tasa de desempleo y su baja tasa de empresas por 1000 habitantes. Así mismo su nivel de crecimiento del PIB es medio y sus inversiones en infraestructura son bajas teniendo en cuenta su territorio. Es la Comunidad más poblada y la segunda más extensa.

Tras el estudio de los resultados de este nuevo clúster, se llega a conclusiones parecidas a las obtenidas en el clúster 2 . El análisis de conglomerados llevado a cabo crea clústers en regiones con situaciones económicas similares que, a su vez, invierten un dinero parecido en función del territorio que ocupan.

\section{CONCLUSIONES.}

El análisis de los resultados obtenidos, tras la realización de la parte metodológica, permite alcanzar diferentes conclusiones.

La primera de ellas es la desigualdad económica existente entre las CCAA españolas. Mientras que existen CCAA que ocupan una posición económica alta, hay otras CCAA cuya situación es francamente comprometida. Las clasificaciones realizadas permiten, grosso modo, dividir la realidad de las regiones en economías con situaciones económicas altas, medias y bajas, tal y como se ha ido realizando a lo largo del artículo.
La crisis financiera que comenzó en el año 2007/2008 ha hecho mella en la realidad económico-social de las CCAA. Eso es visible en las diferencias, tan importantes, de los datos medios usados (desempleo, inversiones, número de empresas y crecimiento del PIB) en los dos periodos utilizados.

Así mismo, los datos demuestran que, efectivamente, España está sufriendo una pequeña aceleración. Durante el periodo 2007-2013, el crecimiento del PIB fue negativo, los datos de desempleo se elevaron y el número de empresas descendió bruscamente. Sin embargo, en el periodo 2014-2015, los datos de crecimiento del PIB fueron, en el caso de todas las CCAA, positivos.

Aun así, todavía es pronto para afirmar que España ha superado la gran crisis de 2007. Este hecho se evidencia en las inversiones en infraestructuras presupuestadas para los años siguientes a los estudiados en este artículo, pues hasta el momento presente no se cuenta con datos oficiales del gasto real. Si bien en 2016 el dinero destinado a infraestructura fue similar al de 2015, en el año 2017 esta suma cayó en picado hasta ser el menor desde el inicio de la crisis ${ }^{20}$. En números relativos, en 2017 el declive de la inversión pública en infraestructura en comparación con el año anterior se sitúa en el 20'6\%, llegando a ser aproximadamente una tercera parte de lo que se invertía en los años anteriores a la crisis ${ }^{21}$. De hecho, únicamente la partida de aeropuertos

20 El gasto en infraestructuras será de 7.540 millones, el menor desde el inicio de la crisis. Diario Público. (4 de Abril de 2017). [Acceso el 5 de abril de 2017] Disponible en http://www.publico.es/economia/inversion-infra estructuras-cae-21-presupuestos.html

21 MUÑOZ R. Presupuestos 2017: la inversión pública en infraestructura cae un 20,6\%. Diario el País. (4 de abril de 2017). [Acceso el 5 de abril de 2017] Disponible en https://elpais.com/economia/2017/04/04/actualidad/ 1491301205_295453.html 
creció, siendo especialmente significativos los desplomes de la inversión en obras hidráulicas (-34\%) y en ferrocarril (-29'2\%). Será de sumo interés realizar un estudio similar al planteado en estas páginas con la inclusión de los datos oficiales de inversión en infraestructura por CCAA en los años posteriores a 2015 .

Así mismo, se debe añadir que la tasa de crecimiento del PIB en España para 2016 y 2017 se situó en el 3,3 y 3,6\% ${ }^{22}$ respectivamente, siendo la tendencia igualmente positiva para los próximos cursos. El desempleo, de igual manera, continuó disminuyendo, colocándose en el 21\% a finales del primer trimestre 2016 y en el $18,75 \%$ a finales del primer trimestre de $2017^{23}$. Además el número de empresas activas aumentó un 1,4\% durante 2016 y se situó en 3,28 millones ${ }^{24}$. Con el respaldo de todos estos datos se puede garantizar que la economía española está creciendo poco a poco, pero aún está lejos de alcanzar los niveles de los años anteriores a la crisis. Por tanto, no se puede afirmar con rotundidad que la crisis del año 2007 ha abandonado por completo el país.

Los resultados obtenidos tras la práctica metodológica arrojan varias ideas. El País Vasco, Madrid, Cataluña y Galicia son las CCAA con mejores situaciones económicas en España tras el periodo 2014-2015, algo que también ocurría en el periodo 20072013. Las economías con situaciones económicas más bajas, en ambos periodos, son Andalucía y Canarias.

22 Datos obtenidos de la web del Instituto Nacional de Estadística de España (2017). [Acceso el 5 de octubre de 2017]. www.ine.es

23 INE (2016-2017). [Acceso el 5 de octubre de 2017] Disponible en http://www.ine.es/jaxiT3/Datos. htm?t=4247

24 El número de empresas activas aumentó un 1,4\% durante 2016 y se situó en 3,28 millones. Instituto Nacional de Estadística. (31 de julio de 2017). [Acceso el 5 de octubre de 2017] Disponible en http://www.ine. es/prensa/dirce_2017.pdf
Otra conclusión a destacar, tras los resultado obtenidos, es la íntima relación existente entre inversión en infraestructura y desarrollo territorial. Eso es palpable, por ejemplo, en términos de inversión en infraestructura en cada uno de los periodos analizados. Mientras que en el periodo anterior a la crisis, las inversiones eran cuantiosas, durante y una vez finalizada la misma, esas inversiones son escasas.

Así mismo, se comprueba que la CCAA con mejor posición económica en el periodo 2014-2015, el País Vasco, es la región que más recursos destina a la inversión por infraestructura tanto por superficie como por habitantes. Además, otras regiones con situaciones económicas altas invierten en infraestructura cantidades medias o superiores a esta.

Canarias, una CCAA con situación económica baja, pretende dejar atrás la crisis financiera, a priori, realizando grandes inversiones en infraestructura. Esto demuestra hasta qué punto, el desarrollo territorial y las inversiones en infraestructura están relacionadas.

La tercera conclusión que se puede alcanzar del análisis realizado es que las CCAA con situaciones económicas similares tienden a invertir niveles parejos es su inversión en infraestructura por superficie. Se ha hablado mucho, a lo largo del artículo, sobre esta idea. La densidad de población de las CCAA, sin duda, juega un papel fundamental en el apartado de inversiones en infraestructura. Se ha podido comprobar, a lo largo del presente artículo, cómo regiones con inversiones altas en infraestructura por habitante, pasan a ser medias o bajas en el caso de sus inversiones en infraestructura por superficie. 
Por tanto, el análisis de conglomerados realizado en función de las inversiones en infraestructura por habitante, genera grupos de CCAA con situación económica diferente, mientras que, en el caso de las inversiones infraestructura por superficie, los conglomerados resultantes clasifican a las CCAA de manera mucho más real en el caso de su situación económica. La situación económica de las CCAA españolas y su inversión en infraestructura por superficie se encuentran íntimamente ligadas.

\section{REFERENCIAS BIBLIOGRÁFICAS}

Aghón, G.; Alburquerque F. y Cortés P. (2001): Desarrollo económico local y descentralización en América Latina: un análisis comparativo, LC/L.1549, Santiago de Chile, Comisión Económica para América Latina y el Caribe (CEPAL).

Alburquerque, F., \& Universidad de Sevilla. Instituto de Desarrollo Regional. (2002). Planes de desarrollo sostenible en los parques naturales de Andalucía: Aproximación conceptual y metodológica. Coria del Rio (Sevilla): Instituto de Desarrollo Regional, Fundación Universitaria.

Convivencia Cívica Catalana (2016): Las inversiones en infraestructura en España. Análisis y distribución territorial. Estudio de la última década (2006-2015).

Diario Público. El gasto en infraestructuras será de 7.540 millones, el menor desde el inicio de la crisis. Diario Público. (4 de Abril de 2017).

Expansión. Diario económico. www.expansion.com/economia/datosmacro.html

Fritz, J., \& Hanus, J. (2015). The European integrated maritime policy: The next five years. Marine Policy, 53, 1-4. doi:10.1016/j.marpol.2014.11.005

González Enciso, A., \& Matías Barco, J. M. (2013). Historia económica de España. Barcelona: Ariel.

González Julián. La inversión en infraestructuras se convierte en enfermedad crónica. Capital Madrid (22 de Agosto de 2016).

Instituto Nacional de Estadística. www.ine.es

Libro verde de transporte y cambio climático (2010). Madrid: Colegio de Ingenieros de Caminos, Canales y Puertos.

Ministerio de Empleo y Seguridad Social. www.empleo.gob.es

Ministerio de Fomento. www.fomento.gob.es

Muñoz R. Presupuestos 2017: la inversión pública en infraestructura cae un 20,6\%. Diario el País. (4 de Abril de 2017).

Peña Sánchez, A. R. (2008). Las disparidades económicas regionales en España: Las infraestructuras como factor de convergencia en el periodo 1980-2000. Revista De Estudios Regionales, (82), 105-132. 
Sánchez Calderón, F. V. (2008). Elementos para una geopolítica de los megaproyectos de infraestructura en América Latina y Colombia. Cuadernos De Geografía,(17), 7-21.

Vázquez Barquero, A. (2009). Desarrollo local, una estrategia para tiempos de crisis. Apuntes Del CENES, 28(47), 117-132.

\section{Para citaciones:}

García-Marquez, F. A. (2017). Aproximación a las inversiones en infraestructura de las Comunidades Autonómas Españolas. Panorama Económico, 25, 3, pp. 311-332.

\section{AUTOR}

Francisco Antonio García-Marquez

Profesor tutor del Centro Asociado de la UNED en Cádiz (España). Actualmente doctorante en Administración de Empresas y Entorno Económico de la Universidad de Cádiz y Magister en En Economía y Desarrollo Territorial de la misma institución 
